\title{
Correction: Reduced-intensity conditioning is effective for hematopoietic stem cell transplantation in young pediatric patients with Diamond-Blackfan anemia
}

Shun Koyamaishi - Takuya Kamio - Akie Kobayashi • Tomohiko Sato • Ko Kudo - Shinya Sasaki • Rika Kanezaki • Daiichiro Hasegawa · Hideki Muramatsu • Yoshiyuki Takahashi • Yoji Sasahara · Hidefumi Hiramatsu • Harumi Kakuda - Miyuki Tanaka - Masataka Ishimura - Masanori Nishi - Akira Ishiguro • Hiromasa Yabe • Takeo Sarashina - Masaki Yamamoto • Yuki Yuza • Nobuyuki Hyakuna • Kenichi Yoshida • Hitoshi Kanno (D) Shouichi Ohga • Akira Ohara • Seiji Kojima - Satoru Miyano • Seishi Ogawa • Tsutomu Toki • Kiminori Terui • Etsuro Ito

(c) The Author(s), under exclusive licence to Springer Nature Limited 2020

Correction to: Bone Marrow Transplantation https://doi.org/10.1038/s41409-020-01056-1

Published online 18 September 2020
In the original version of this Article, in Table 1 the Year of HST for cases 3, 4, 15, and 25 was not shown. They now appear in Table 1 in the PDF and HTML versions of the Article. 


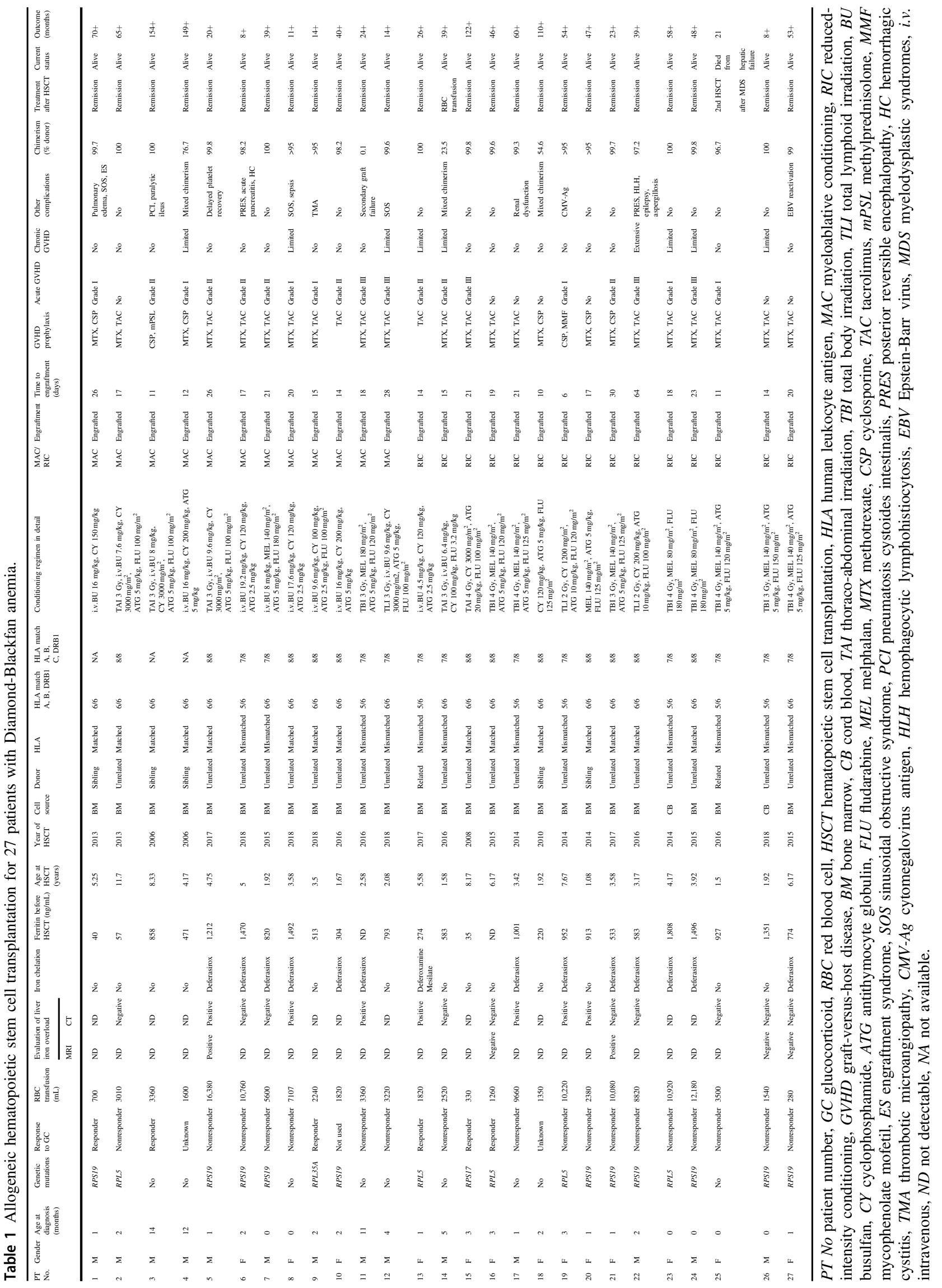

